\title{
LEISHMANIOSI VISCERALE: \\ ANALISI RETROSPETTIVA DEI CASI OSSERVATI NEGLI ULTIMI TRE ANNI
}

\author{
Fumarola L., Monno R., Bellomo G., Rizzo C., De Vito D, \\ Rizzo G. \\ Dipartimento di Medicina Interna e Medicina Pubblica, Sezione \\ di Igiene, Policlinico, Università degli Studi di Bari, \\ Piazza G. Cesare 1/, 701 24, Bari
}

Introduzione. La leishmaniosi viscerale (LV) causata da Leishmania infantum è endemica nel Sud Italia. Nel periodo agosto 2002, maggio 2005 presso il Laboratorio di Microbiologia e Parassitologia, U.O. Igiene e Sanità Pubblica I, Policlinico, Bari, sono stati esaminati 319 sieri di pazienti con sospetto di LV.

Metodi. L'indagine di laboratorio è stata effettuata mediante ricerca in immunofluorescenza di anticorpi totali nel siero (Leishmania-Spot IF, bioMérieux, Italia) e/o osservazione microscopica di biopsie midollari.

Risultati. Nove pazienti (6 soggetti adulti e 3 pediatrici) avevano titoli anticorpali indicativi di infezione in atto. Di questi, 3 risiedevano nella provincia di Bari, 2 in quella di Taranto, 2 in quella di Brindisi, 1 in provincia di Matera e 1 in quella di Cosenza.

In 7 pazienti furono evidenziati amastigoti nell'aspirato midollare. Il rapporto $\mathrm{M} / \mathrm{F}$ era $5 / 4$. Segni clinici al ricovero furono: febbre con brividi scuotenti nel $66.6 \%$ dei casi, epatomegalia $(77.7 \%)$, splenomegalia $(88.8 \%)$, neutropenia $(44.4 \%)$, piastrinopenia $(66.6 \%)$, leucopenia $(55.5 \%)$ ed anemia (44.4\%). Pancitopenia fu riscontrata solo in 1 bambino.Due pazienti risultarono HIV/HCV positivi.

Due pazienti furono trattati con meglumina stibiato, 4 con amfotericina B liposomiale, 2 con amfotericina seguita da meglumina. In 3 casi si rese necessaria la trasfusione.

Non sono state osservate ricadute nei pazienti ritornati al controllo.

Conclusioni. Il numero dei casi diagnosticati nel periodo studiato $(2.82 \%)$ è risultato inferiore a quello da noi riscontrato nel precedente triennio (1999-2002) e risultato pari al $7.2 \%$. Rimane da stabilire se tale decremento sia da ascrivere ad una maggiore disponibilità sul territorio di idonei strumenti diagnostici o ad un'effettiva riduzione dei casi. Nel periodo 2000-2004 sono stati notificati in Puglia 50 casi di LV e 10 casi di leishmaniosi cutanea con un picco nel 2000 (14 casi) e nel 2001 (16 casi). 